\title{
BALANÇO HÍDRICO E DE ENERGIA PARA PLANTIOS DE EUCALIPTO COM COBERTURA PARCIAL DO SOLO
}

\section{BALANCE OF WATER AND ENERGY FOR EUCALYPTUS PLANTATIONS WITH PARTIAL SOIL COVER}

\author{
Mariana Gonçalves dos Reis ${ }^{1}$ Aristides Ribeiro ${ }^{2}$ Raquel Couto Evangelista Baesso ${ }^{3}$ \\ Wesley Gonçalves de Souza ${ }^{4}$ Sebastião Fonseca ${ }^{5}$ Rodolfo Araujo Loos ${ }^{6}$
}

\section{RESUMO}

Plantios com idade inicial de desenvolvimento apresentam descontinuidade na cobertura do solo, tendendo a apresentar maior exposição do dossel ao vento e à radiação solar, o que altera as interações solo-plantaatmosfera. O objetivo deste trabalho foi estudar os componentes dos balanços hídricos e de energia ao longo do primeiro ano de desenvolvimento do eucalipto na região da planície costeira brasileira. O sítio experimental está localizado em uma área pertencente à empresa Fibria Celulose no município de Aracruz - ES. O espaçamento entre as árvores do plantio de eucalipto da área estudada foi de $3 \times 3 \mathrm{~m}$ e a data do plantio foi em 15/08/2004. O período de estudo foi compreendido entre a data do plantio até a cultura ter completado 19 meses de idade. Verificou-se maior disponibilidade de energia no período de verão e que a precipitação influenciou diretamente na partição do balanço de energia, sendo que, durante todo o período estudado, a fração da energia disponível destinada à evapotranspiração foi sempre maior que a fração destinada ao aquecimento do sistema solo-planta-atmosfera, apresentando uma relação de 59,57\% de $\lambda E / R_{n}$. Observou-se também que o balanço hídrico com a evapotranspiração modelada apresentou boa correspondência ao comportamento da umidade observada, apresentando um coeficiente de determinação de 0,94. Os maiores portes das árvores, maiores índices de área foliar e sistema radicular, favoreceu a evapotranspiração, mostrando que a maior parte da energia disponível foi utilizada para mudança de fase da água.

Palavras-chave: plantio jovem de eucalipto; disponibilidade de energia e evapotranspiração.

\footnotetext{
ABSTRACT

Eucalyptus plots with initial development ages presented discontinuity in soil cover, resulting in greater exposure of the leaves to wind and solar radiation, which alters soil-plant-atmosphere interactions. The objective of this study was to study the components of the water and energy balances along the first year of eucalyptus development in the Brazilian coastal plain region. The experimental site is located in an area belonging to the company Fibria in the municipality of Aracruz, Espírito Santo state, Brazil. The space between the planted eucalyptus trees in the area studied was $3 \times 3 \mathrm{~m}$ and the data of planting was on August $15^{\text {th }}, 2004$. The period of study lasted from the planting date until the plot reached an age of 19 months. It was verified that there was a greater availability of energy during the summer and the precipitation directly influenced the energy balance where during the period of study the energy available

1 Graduanda do curso de Agronomia, Universidade Federal de Viçosa,UFV, CEP 36570-977, Fone: (0xx31) 3899 1906, Viçosa (MG), Brasil. mariana.reis@ufv.br

2 Engenheiro Agrônomo, Doutor, Professor do Departamento de Engenharia Agrícola, Universidade Federal de Viçosa, UFV, CEP 36570-977, Viçosa (MG), Brasil. ribeiro@ufv.br

3 Engenheira Agrícola e Ambiental, Doutora pela Universidade Federal de Viçosa, UFV, CEP 36570-977, Viçosa (MG), Brasil. evangelista.raquel@ufv.br

4 Engenheiro Agrimensor, Msc., Pesquisador da Empresa Ouro Verde Agrosilvopastoril Ltda., CEP 69301-041, Boa Vista (RR), Brasil.wesley_ibnv@hotmail.com

5 Engenheiro Florestal, Msc., Pesquisador da Empresa Fibria Celulose S/A, Rod. Aracruz, Barra do Riacho, Km 25, Caixa Postal 331011, CEP 29197-000, Aracruz (ES), Brasil. sf@fibria.com.br

6 Engenheiro Agrônomo, Dr., Pesquisador da Empresa Fibria Celulose S/A, Rod. Aracruz, Barra do Riacho, Km 25, Caixa Postal 331011, CEP 29197-000, Aracruz (ES), Brasil. raloos@fibria.com.br

Recebido para publicação em 4/02/2011 e aceito em 4/10/2012
} 
necessary for evapotranspiration was always greater than the fraction necessary for heating the soil-plantatmosphere system, presenting a $\lambda E / \mathrm{R}_{\mathrm{n}}$ ratio of $59.57 \%$. It was also observed that the water balance with the modeled evapotranspiration showed a good correspondence with the observed moisture content, presenting a determination coefficient of 0,94 . In the majority of trees, greater indices of leaf and root system areas favored evapotranspiration, indicating that most energy available was utilized for changing the phase of water.

Keywords: young eucalyptus plantation; availability of energy and evapotranspiration.

\section{INTRODUÇÃO}

Vários trabalhos têm se dedicado a estimar os balanços de água e energia para espécies de eucalipto (SILBERSTEIN et al., 2001; ALMEIDA et al., 2007; HUBBARD et al., 2010; CABRAL et al., 2010; FORRESTER et al., 2010; YUNUSA et al., 2010), contudo poucos são aqueles que se dedicam a entender esses processos para plantios jovens com cobertura parcial do solo.

Dentre as florestas plantadas no Brasil verifica-se destaque para a espécie de eucalipto que atualmente conta com uma área plantada de quatro milhões e meio de hectares. Somente entre os anos de 2004-2009, se observou uma expansão de mais de um milhão de hectares de área plantada com eucalipto (crescimento acumulado de $41,1 \%$ no período) (ABRAF, 2010).

O predomínio dos plantios de eucalipto no Brasil se deve por esta espécie silvicultural ser de crescimento rápido e alta produtividade. Aliado a isso, verifica-se aumento de novos investimentos de capitais nacionais e internacionais, bem como a expansão de empresas demandantes desta matéria-prima para processos industriais no país.

Contudo, o reflorestamento com árvores de eucalipto tem causado grande preocupação ambiental no que diz respeito às implicações desta mudança de uso da terra nos balanços hídrico e de energia, por não se saber os impactos de plantios de extensivas áreas no clima regional e global. Segundo Almeida et al. (2010), a expansão das plantações de eucalipto gera grande preocupação sobre seus efeitos nos recursos hídricos locais.

Sabe-se que o crescimento e o desenvolvimento dos vegetais é consequência de vários processos fisiológicos controlados pelas condições ambientais e características genéticas de cada espécie vegetal. O movimento estomático é o principal mecanismo fisiológico que controla as trocas gasosas nas plantas terrestres (MIELKE et al., 1999). Portanto, para melhor compreender o crescimento, o desenvolvimento e o impacto hidrológico de uma plantação de eucalipto, faz-se necessário conhecer os fatores que controlam o uso da água.

Vários fatores ambientais influenciam a abertura e o fechamento estomático, determinando a maior ou a menor transferência de vapor de água para a atmosfera (SOUZA, 2006). Dentre esses, destacam-se os níveis de umidade do solo, o teor de umidade do ar, radiação solar, temperatura do ar e déficit de pressão de vapor d'água. Corroborando com Zanon e Finger (2010), fenômenos meteorológicos como precipitação, temperatura e luz afetam o desenvolvimento das árvores.

Normalmente, os modelos de crescimento consideram na estimativa da condutância estomática, o dossel como sendo uma folha grande, única e contínua (teoria do "Big Leaf"). Esta aproximação não é adequada na etapa inicial de desenvolvimento de florestas. Esta idade em relação às outras tem uma grande importância, sendo que nesse período as árvores apresentam maior taxa de crescimento, uma vez que o ganho de biomassa está diretamente relacionado aos processos de evapotranspiração (perda de vapor d'água) e fotossíntese (ganho de $\mathrm{CO}_{2}$ ).

Neste contexto, este estudo teve como objetivo quantificar os componentes dos balanços hídrico e de energia ao longo do primeiro ano de desenvolvimento do eucalipto na região da planície costeira brasileira.

\section{MATERIAL E MÉTODOS}

\section{Caracterização do local}

Os estudos foram conduzidos no sítio experimental, pertencente à empresa Fibria Celulose no município de Aracruz - ES, cujas coordenadas geográficas limítrofes são: latitude de 1951'16" a $19^{\circ} 52^{\prime} 18^{\prime \prime}$ S e longitude de $40^{\circ} 13^{\prime} 15^{\prime \prime}$ a $40^{\circ} 11^{\prime} 44^{\prime \prime}$ W. A área total do projeto é de 286,12 ha, sendo 188,69 ha plantados com Eucalyptus grandis, 85,44 ha de floresta nativa e 11,99 ha equivalentes às estradas. 
O clima da região é Aw, segundo a classificação de Köppen, com temperatura média anual de $23^{\circ} \mathrm{C}$ e precipitação média anual de $1200 \mathrm{~mm}$. Os tipos de solos existentes na região são originados de sedimentos da Formação Barreiras, os quais são: Argissolo Amarelo textura médio-argilosa (PA1), Plintossolo Háplico (FX) e Argissolo Amarelo moderadamente rochoso (PA8). O espaçamento entre as árvores do plantio de eucalipto da área estudada foi de 3 × 3 m e a data do plantio foi em 15/08/2004. $\mathrm{O}$ período de estudo foi compreendido entre a data do plantio até a cultura ter completado 19 meses de idade.

\section{Dados Meteorológicos}

Os dados meteorológicos (Tabela 1) necessários para o desenvolvimento do trabalho, bem como para a alimentação dos modelos, foram coletados por sensores instalados em uma torre de $38 \mathrm{~m}$ de altura, sendo esta disposta entre quatro árvores, obedecendo ao espaçamento do plantio no sítio experimental. Monitorou-se a variação da umidade do solo em seis profundidades distintas, as quais foram de: $0-15,15-30,30-45,45-75,75-105$ e $105-135 \mathrm{~cm}$, com um perfilador de umidade do solo. As características físicas do solo foram determinadas pelas análises densidade aparente, capacidade de campo e ponto de murcha permanente.

\section{Área Foliar}

Escolheram-se quatro árvores dentro do sítio experimental que fossem representativas, para a análise em laboratório da largura, comprimento e área foliar, sendo este o método destrutivo utiliza- do para medição e estimativa do índice de área foliar (IAF). Para isso, a partir do inventário florestal da empresa, calculou-se a altura média e a circunferência a altura do peito (CAP) médio com base em todas as árvores delimitadas pelo sítio experimental, identificando em seguida uma árvore cuja altura e CAP fossem semelhantes aos valores médios calculados. Dividiu-se a árvore em três terços (inferior, médio e superior), medindo o diâmetro da copa em cada um dos terços. Foi obtido o peso seco de 150 folhas coletadas aleatoriamente ao longo de toda árvore. A partir da área foliar média e do peso seco das 150 folhas, ambos determinados em laboratório, foi possível calcular a área foliar média total de uma árvore, uma vez que também foi calculado o peso seco total de folhas para outra árvore dentro do mesmo sítio experimental. A projeção média da copa sobre o terreno foi calculada a partir dos valores medidos do diâmetro do terço inferior das árvores.

\section{Modelagem da Evapotranspiração}

\section{Método da Razão de Bowen}

A razão entre os calores sensível e latente foi proposta por Bowen, em 1926, como forma de estudar a partição da energia disponível, isto é:

$$
\beta=\frac{\lambda E}{H}
$$

Em que: $\beta$ é a razão de Bowen; $\lambda$ E é o calor latente, $\mathrm{W} \mathrm{m}^{-2}$; e $\mathrm{H}$ é o calor sensível, $\mathrm{W} \mathrm{m}^{-2}$. $\mathrm{O}$ valor de $\beta$ depende fundamentalmente das condições hídricas da superfície evaporante. Se a superfície estiver umedecida, maior parte do saldo de radia-

TABELA 1: Especificações dos sensores utilizados para medição das variáveis ambientais no município de Aracruz - ES.

TABLE 1: Specifications of the sensors used for measuring the environmental variables in the municipality of Aracruz, ES state.

\begin{tabular}{lll}
\hline Sensor & Modelo & Marca \\
\hline Temperatura e umidade do ar & HMP45C & Vaisala, Vantaa, Finland \\
Saldo radiômetro & CNR1 & Kipp \& Zonen, Delft, The Netherlands \\
Pluviômetro & TB4 (CSI) & Hydrological Services Pty. Ltd., Sydney, Australia \\
Anemômetro & 03001 & R.M. Young Company, Traverse, USA \\
Fluxo de calor no solo & HFT3 & Campbell Scientific Instruments, Utah, USA \\
Umidade do solo & Diviner 2000 & Sentek Pty Ltd, Stepney, Australia \\
\hline
\end{tabular}


ção $\left(R_{n}\right)$ será utilizada em $\lambda E$, resultando em um $\beta$ pequeno. Se, pelo contrário, a superfície evaporante apresentar restrições hídricas, maior parte de $\mathrm{R}_{\mathrm{n}}$ será utilizada no aquecimento do ar, resultando em um $\beta$ elevado.

Com a razão de Bowen, a equação do balanço de energia pode ser escrita da seguinte forma:

$$
\lambda E=\frac{R_{n}-G}{1+\beta}, \text { para } \beta \neq-1
$$

Em que: $\mathrm{R}_{\mathrm{n}}$ é o saldo de radiação, $\mathrm{W} \mathrm{m}^{-2}$; e $\mathrm{G}$ é o fluxo de calor no solo, $\mathrm{W} \mathrm{m}^{-2}$.

A partir dos valores medidos em campo de fluxo de calor no solo, foi possível obter os valores de fluxo para todo período estudado pela seguinte equação:

$$
G=0,0072 R_{n}-0,2392
$$

Em que: $\mathrm{R}_{\mathrm{n}}$ é o saldo de radiação, $\mathrm{W} \mathrm{m}^{-2}$.

De acordo com o método aerodinâmico podemos estimar a evapotranspiração (ET) pela equação a seguir:

$$
\lambda E=-K_{e} \rho \lambda \frac{\delta q}{\delta z}
$$

analogamente,

$$
H=-K_{H} c_{p} \frac{\delta \theta}{\delta z}
$$

Em que: $K_{e}$ é o coeficiente de transferência turbulenta de vapor de água; $\rho$ é a massa específica do ar, $\mathrm{kg} \mathrm{m}^{-3} ; \lambda$ é o calor latente de vaporização, $2,45 \mathrm{MJ} \mathrm{kg}^{-1} ; \delta \mathrm{q} / \delta \mathrm{z}$ é o gradiente vertical de umidade, $\mathrm{kg} \mathrm{kg}^{-1} ; K_{H}$ é o coeficiente de transferência turbulenta do calor sensível; $c_{p}$ é o calor específico do ar a pressão constante $1,013 \mathrm{MJ} \mathrm{kg}^{-1}{ }^{\circ} \mathrm{C}^{-1} \mathrm{e} \delta \theta / \delta \mathrm{z}$ é o gradiente vertical de temperatura do ar, ${ }^{\circ} \mathrm{C}$.

Segundo Munro (1985) e Denmead e Bradley (1985), os coeficientes de transferência turbulenta do calor sensível e vapor d'água, $\mathrm{K}_{\mathrm{H}}$ e $\mathrm{K}_{\mathrm{e}}$ podem ser considerados iguais acima de florestas:

$$
\beta=\frac{c_{p} \delta \theta}{\lambda \delta q}=\frac{c_{p}\left(\theta_{2-} \theta_{1}\right)}{\lambda\left(q_{2-} q_{1}\right)}
$$

\section{Modelagem da Evapotranspiração em Plantios Esparsos}

\section{Transpiração}

Para estimativa da transpiração, pela equação de Penman-Monteith, levou-se em consideração as adaptações que serão mostradas a seguir e que resultaram na expressão abaixo:

$$
\lambda E=\frac{\Delta \cdot R_{n f}+\eta \rho c_{p} \frac{D_{a}}{r_{a}}}{\Delta+\eta \gamma\left(j+\frac{r_{f}}{r_{a}}\right)}
$$

onde, $\Delta$ é a declividade da curva de pressão de vapor, $\mathrm{kPa}^{\circ} \mathrm{C}^{-1} ; \mathrm{R}_{\mathrm{nf}}$ é o saldo de radiação nas folhas, $\mathrm{W} \mathrm{m}^{-2}, \gamma$ é o coeficiente psicrométrico, $\mathrm{kPa}^{\circ} \mathrm{C}^{-}$ ${ }^{1}$; $\mathrm{r}_{\mathrm{f}}$ é a resistência estomática, $\mathrm{s} \mathrm{m}^{-1}, \mathrm{r}_{\mathrm{a}}$ é a resistência aerodinâmica, $\mathrm{s} \mathrm{m}^{-1}$; Da é o déficit de saturação do vapor d' água médio do dia, $\mathrm{kPa} ; \mathrm{j}$ depende da espécie da folha, sendo $\mathrm{j}=1$ para folhas anfiestomáticas (estômatos nas duas faces da folha) e $\mathrm{j}=2$ para folhas hipoestomáticas (estômatos apenas na face inferior da folha); $\eta$ é o fator originário do desenvolvimento teórico da equação ao se adotar aproximação de que a relação entre as resistências da camada limite ao transporte de vapor d'água e ao de calor sensível é próxima da unidade, ou seja, $\eta=0,93$;

\section{Saldo da Radiação}

O saldo de radiação no dossel vegetativo em plantios esparsos pode ser obtido pela equação a seguir, proposta e validada por Riou et al. (1989):

$$
R_{n f}=\frac{R_{n} R_{g a}}{R_{g}(1-r)}
$$

Em que: $\mathrm{R}_{\mathrm{nf}}$ é o saldo de radiação nas folhas, $\mathrm{W} \mathrm{m}^{-2} ; \mathrm{R}_{\mathrm{ga}}$ é a radiação interceptada pelo dossel, $\mathrm{W} \mathrm{m}^{-2} ; \mathrm{R}_{\mathrm{n}}$ é a irradiância solar global medida, $\mathrm{W}$ $\mathrm{m}^{-2}$; e r é o albedo.

A radiação interceptada pelo dossel foi calculada como sendo a diferença entre a irradiância solar global que atinge o dossel e a porção desta radiação que atinge o solo, sendo esta última descrita pela Lei de Beer-Bouguer-Lambert:

$$
R_{g a}=R_{g}-R_{g}\left(1-\ell^{-K I A F}\right)
$$

Em que: $k$ é o coeficiente de extinção, 0,40 ; e IAF é o índice de área foliar, $\mathrm{m}^{2} \mathrm{~m}^{-2}$.

O albedo foi calculado a partir dos dados de radiação de onda curta incidente e refletida, coletados na torre de observação meteorológica com o sensor saldo radiômetro.

\section{Resistência Aerodinâmica}

Em plantios esparsos, a caracterização do comprimento da rugosidade e o deslocamento do 
plano zero, utilizados para o cálculo da resistência aerodinâmica, fica prejudicada pela dificuldade de desenvolvimento da camada limite característica nesta situação, devido a uma parte das folhas estarem mais expostas ao vento do que outras.

Nestas condições, a resistência aerodinâmica pode ser estimada através da equação desenvolvida por Landsberg e Powell (1973) em trabalhos com árvores isoladas, ou seja,

$$
r_{a}=58 p^{0,56}\left(\frac{d^{\prime}}{u}\right)^{0,5}
$$

Em que: $p$ é a medida da razão da área foliar total da árvore e a área da folhagem projetada num plano vertical, $\mathrm{m} ; u$ é a velocidade do vento, $\mathrm{m} \mathrm{s}^{-1} ; \mathrm{d}^{\text {' }}$ é a dimensão característica das folhas, $m$.

O valor de $d$ 'é admitido por alguns autores como sendo a largura ou o comprimento das folhas, neste trabalho foi adotado $d$ 'como sendo a raiz quadrada da área foliar.

\section{Resistência Estomática}

Para o cálculo da resistência estomática utilizou-se a equação proposta por Thorpe et al. (1980), ou seja,

$$
r_{f}=\frac{1}{g_{r}}\left(\frac{1+\frac{b}{R F A_{f}}}{1-a D P V}\right)
$$

Em que: DPV é o déficit de pressão de vapor d' água, $\mathrm{kPa}$; $\mathrm{g}_{\mathrm{r}}$ é a condutância de referência, $10 \mathrm{~mm} \mathrm{~s}^{-1} ; \mathrm{RFA}_{\mathrm{f}}$ é a radiação fotossinteticamente ativa nas folhas, $\mu \mathrm{mol} \mathrm{m}{ }^{-2} ; a$ e $b$ são coeficientes de ajuste, os quais para este trabalho foram respectivamente, $0,25 \mathrm{kPa}^{\circ} \mathrm{C}^{-1}$ e $69 \mathrm{mmol} \mathrm{m}{ }^{-2}$.

A radiação fotossinteticamente ativa nas folhas, $R F A_{\rho}$ foi calculada através da seguinte expressão:

$$
R A F_{f}=2,32 R_{g a} \frac{A_{f}}{A_{t}}
$$

Em que: $\mathrm{A}_{\mathrm{f}}$ é a área foliar média de cada árvore, $\mathrm{m}^{2}$; e At é a área média da projeção da copa sobre o terreno, $\mathrm{m}^{2}$. O fator 2,32 é usado para transformar $\mathrm{W} \mathrm{m}^{-2} \mathrm{em} \mu \mathrm{mol} \mathrm{m} \mathrm{m}^{-2}$.

\section{Balanço Hídrico}

O balanço hídrico resulta da aplicação do princípio de conservação de massa para a água num volume de solo vegetado. A variação do armazenamento $(\triangle \mathrm{ARM})$, num intervalo de tempo, representa o balanço entre entradas e saídas de água do volume de controle.

Para a modelagem do balanço hídrico consideraram-se as seguintes equações:

$$
\begin{gathered}
A M R_{i}=A M R_{i-1}+P R P_{i}-E T_{i} \\
E T_{i}=T_{i}-I T_{i} \\
I T_{i}=0,1 * P R P_{i}
\end{gathered}
$$

Em que: $A M R_{i}$ é o armazenamento de água na camada das raízes do solo $\mathrm{mm} \mathrm{h}^{-1} ; A M R_{i-1}$ é o armazenamento no período anterior $\mathrm{mm} \mathrm{h}^{-1} ; P R P_{i}$ é a precipitação, mm; $E T_{i}$ é a evapotranspiração, $\mathrm{mm}$ $\mathrm{h}^{-1} ; T_{i}$ é a transpiração das árvores, $\mathrm{mm} \mathrm{h}^{-1}$ e $I T_{i}$ é a interceptação do dossel, mm.

Sabe-se que a quantidade máxima de água retida no solo, contra a força da gravidade, é dada por:

$$
C A D_{i}=(C C-P M) d_{a} z_{i} 10
$$

Em que: $C A D_{i}$ é a capacidade de água disponível, $\mathrm{mm}$; $C C$ é a capacidade de campo, $\%$ em peso; $P M$ é o ponto de murcha permanente, $\%$ em peso; $d_{a}$ é a densidade aparente do solo, $\mathrm{g} \mathrm{cm}^{-3} ; z_{i}$ é a profundidade do sistema radicular, $\mathrm{m}$;

Para determinar a variação em profundidade do sistema radicular do eucalipto, m, utilizou o seguinte modelo:

$$
\begin{array}{ll}
z_{i}=0,002782660439 \mathrm{DAP}+0,226451677452 & . \\
z_{i}=1,60 & \mathrm{DAP} \leq 500 \\
& \mathrm{DAP}>500
\end{array}
$$

Sendo que: DAP são os dias após o plantio, dias.

Para as plantas de eucalipto a profundidade do sistema radicular em média atinge um valor máximo de 1,60 metros.

Para valores de ARM $<0$ foi considerado como igual a 0 , uma vez que indica que o potencial hídrico do solo está no ponto de murcha permanente, não sendo, portanto possível para a planta retirar mais água do solo. Para valores de ARM $>$ CAD considerou-se como igual ao valor de CAD, uma vez que o solo somente consegue reter água na capacidade de campo, sendo o excedente drenado em profundidade para fora da camada das raízes.

\section{RESULTADOS E DISCUSSÃO}

A Figura 1 apresenta a correlação entre os valores de ET modelada com os obtidos pelo método da Razão de Bowen. Nota-se que o coeficiente li- 
near é próximo de 1,0 e o coeficiente angular médio próximo de 0,0 , contudo, verifica-se maior dispersão para maiores valores de ET, sendo estes melhor ajustados para os menores valores. Provavelmente, esse comportamento se deve à maior variabilidade observada no momento em que o dossel se encontrava com maior teor de água livre em função da precipitação.

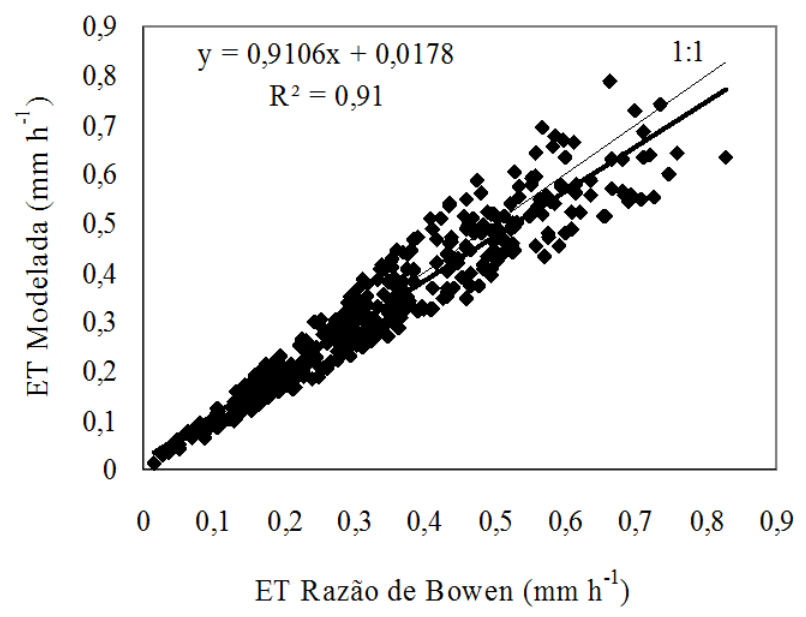

FIGURA 1: Correlação entre totais horários de evapotranspiração modelada e a evapotranspiração obtida pelo método da Razão de Bowen.

FIGURE 1: Correlation between the total hours of evapotranspiration modeled and the evapotranspiration obtained by Bowen's ratio method.
A Figura 2 mostra a variação dos totais mensais de PRP ao longo do experimento, sendo também apresentados os totais de ET modelados.

Os menores valores da ET foram verificados no início do plantio, em que o solo encontrava-se em sua máxima descontinuidade da cobertura vegetal. Também se verificou uma diminuição da ET exatamente um ano depois do plantio, devido ao início do período seco. À medida que se passam os meses, observa-se um aumento da ET, uma vez que maior porte das árvores, maiores índices de área foliar, sistema radicular e maior disponibilidade de água no solo, favorecem a ET. Os valores de PRP influenciam diretamente na partição de energia, o que justifica maior energia destinada para o fluxo de calor latente com o aumento da disponibilidade de água no sistema. Cabral et al. (2010), em seu trabalho com plantio de híbridos de eucalipto (Eucalyptus grandis $\mathrm{x}$ urophylla) no sudeste Brasileiro, verificaram menores valores de ET durante o período de inverno, tendo como causa quase 3 meses contínuos sem chuva.

É importante quantificar o IAF, assim como a sua variação ao longo de seu ciclo produtivo, uma vez que, pelo modelo proposto, a energia disponível para a mudança de fase da água no processo transpirativo depende da extinção da radiação solar pelo dossel vegetativo. Segundo Xavier et al. (2002), a utilização do IAF como um descritor integrado da estrutura do dossel parece ser um bom compromisso, sobretudo se o objetivo de análise são as par-

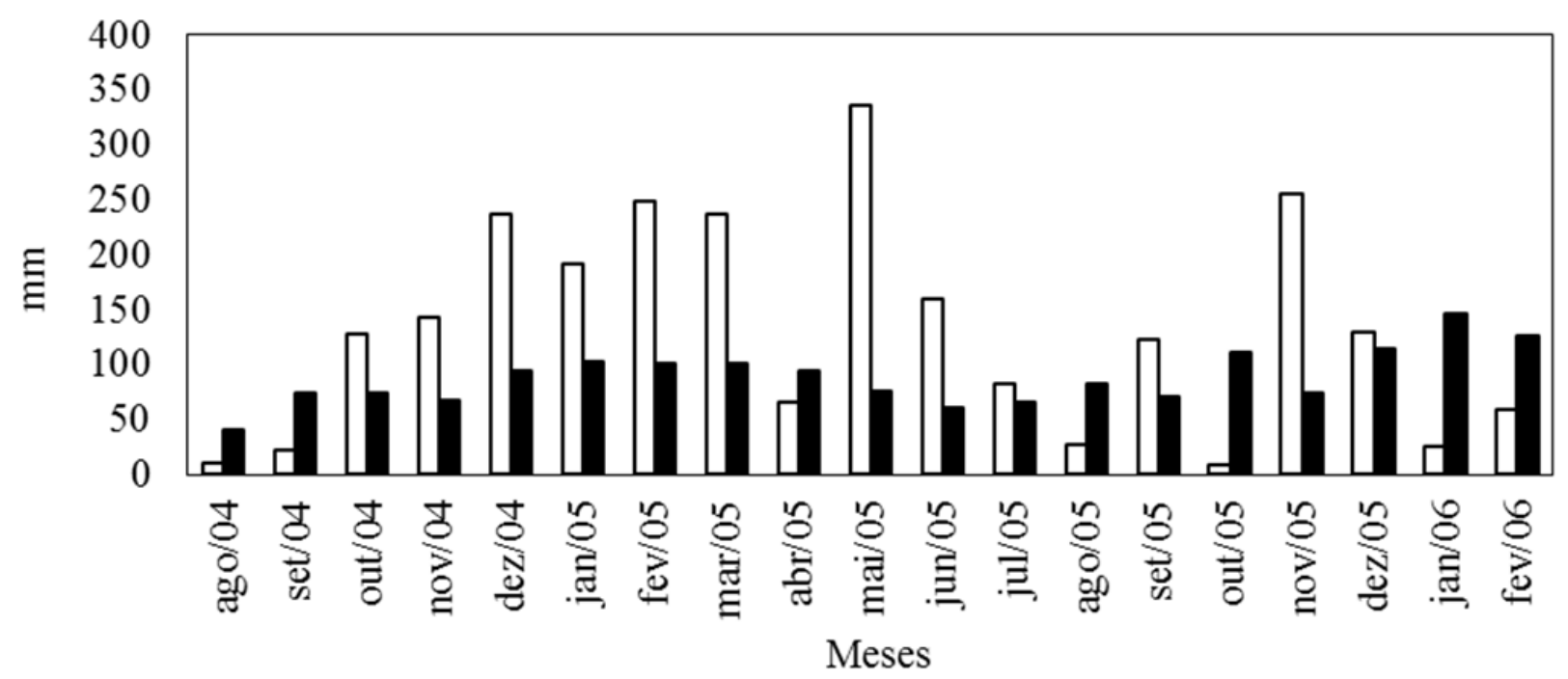

\section{QPRP $\square \mathrm{ET}$}

FIGURA 2: Variação dos totais mensais de precipitação e evapotranspiração modelada.

FIGURE 2: Variation of the monthly totals of precipitation and modeled evapotranspiration. 
tições de energia e de momento entre o dossel e o solo. Assim, quanto maior o IAF maior a energia disponível para calor latente. A Figura 3 mostra o comportamento da curva do IAF ao longo dos meses estudados.

O maior valor de IAF observado neste estudo foi de $4,65 \mathrm{~m}^{2} \mathrm{~m}^{-2}$ no plantio com 19 meses de idade. Xavier et al. (2002) observaram em seu trabalho o maior valor de IAF de 4,31 em clone de eucalipto com 24 meses de idade. Dye (1996) encontrou valores de IAF de aproximadamente 3,0 em plantios de (Eucalyptus grandis) com 3 anos de idade na África do Sul. Cabral et al. (2010) observaram em clones de (Eucalyptus grandis $x$ Eucalyptus urophylla) com 2 anos de idade valores de IAF de 3,4. O'Grady et al. (2008) observaram em lotes de (Eucalyptus globulus) valor de IAF de 3,0, com 2,5 anos de idade. Embora a representação da variação do IAF para todo o ciclo do eucalipto seja exponencial, o crescimento nesta fase inicial de desenvolvimento é linear, o que justifica a escolha do modelo uitlizado neste estudo.

O conhecimento do saldo de energia para florestas, dado pelo balanço de radiação no dossel vegetativo, é de suma importância, tanto para melhor estimar a ET, como para conhecer a quantidade disponível para a biossíntese. A Figura 4 apresenta o $\mathrm{R}_{\mathrm{n}}$ médio diário para o período estudado.

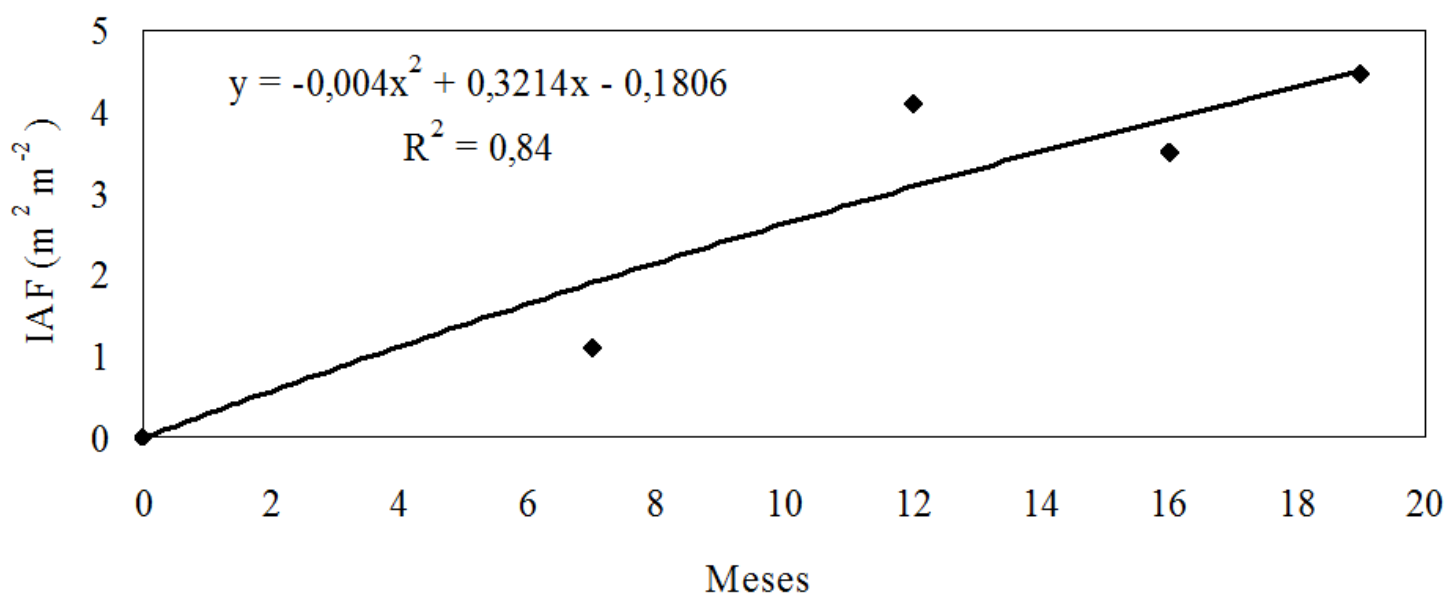

FIGURA 3: Variação do índice de área foliar ao longo do crescimento vegetativo.

FIGURE 3: Variation of the leaf area index during vegetative growth.

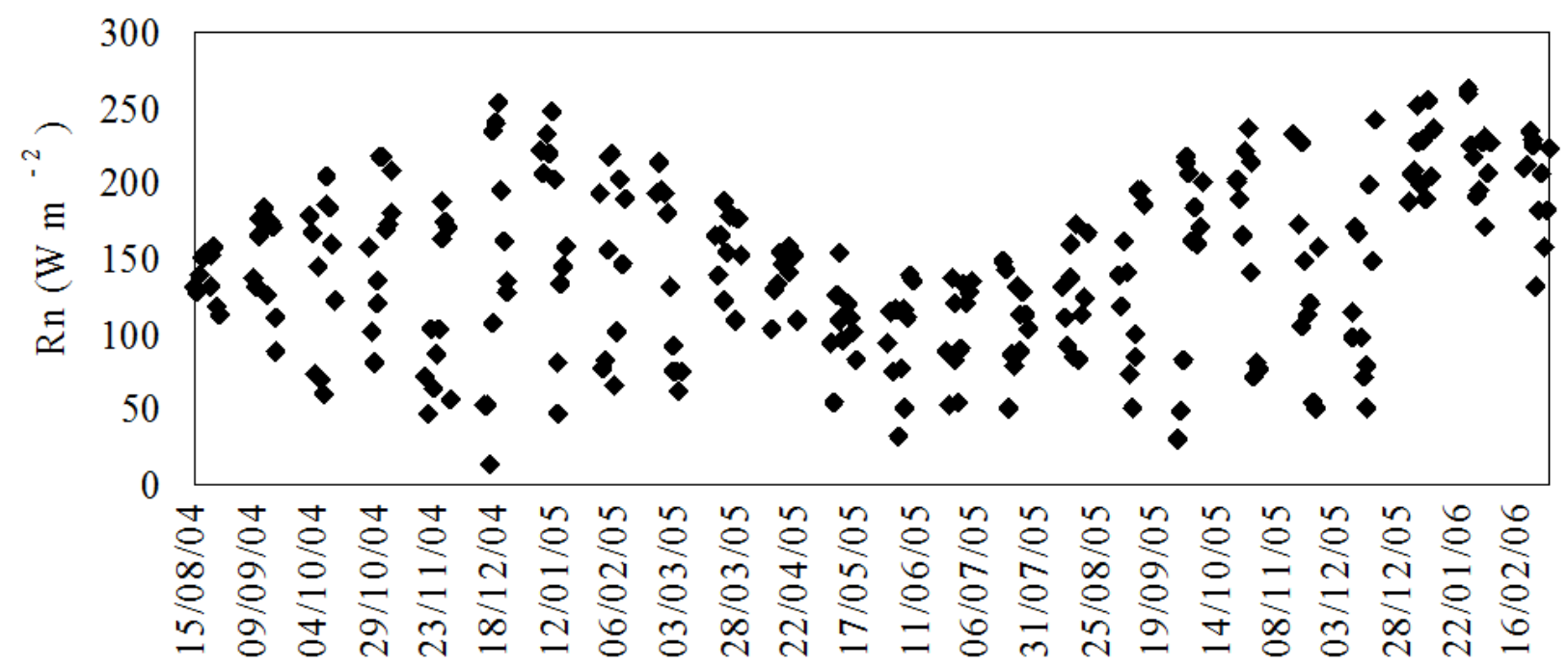

FIGURA 4: Variação diária do saldo de radiação.

FIGURE 4: Variation of the daily total radiation. 
Verifica-se maior disponibilidade de energia no período de verão. Contudo, nesta época do ano, a maior nebulosidade promove uma maior variabilidade diária no $\mathrm{R}_{\mathrm{n}}$, quando se compara com o período de inverno. $\mathrm{O}$ fato da área foliar da cultura crescer proporcionalmente na área de projeção do dossel sobre o terreno explica a interceptação da radiação líquida nas folhas, sendo que, a radiação interceptada pelo dossel, para o inicio e final do período estudado, foi de aproximadamente $55 \%$ da radiação solar global com um albedo médio de $17 \%$.

A Figura 5 mostra a partição do saldo de radiação percentual média para os fluxos de calor no solo $\left(G / R_{n}\right)$, calor latente $\left(\lambda E / R_{n}\right)$ e calor sensível $\left(\mathrm{H} / \mathrm{R}_{\mathrm{n}}\right)$.

A fração da $\mathrm{R}_{\mathrm{n}}$ utilizada para o aquecimento do solo permaneceu constante para todo o período estudado, isso pode ser explicado pelo modelo utilizado para a estimativa ter como base mensal de tempo. Observa-se que a fração da $\mathrm{R}_{\mathrm{n}}$ destinada à ET foi sempre maior que a fração destinada ao aquecimento do sistema solo-planta-atmosfera, mesmo para o período menos chuvoso do ano. Observa-se ainda, que uma maior proporção da $\mathrm{R}_{\mathrm{n}}$ é utilizada para mudança de fase da água no final do período estudado 59,6\% de $\mathrm{R}_{\mathrm{n}}$, em comparação ao inicio do período $50,5 \%$ de $R_{n}$, podendo ser explicado pelo maior porte das árvores e pelo maior volume de solo explorado pelo sistema radicular.

As principais variáveis preditivas do modelo do balanço hídrico foram: a estimativa da ET e a variação da CAD pela simulação do crescimento das raízes. A Figura 6 apresenta variação mensal da CAD, ARM, ET e PRP para todo o período estudado.

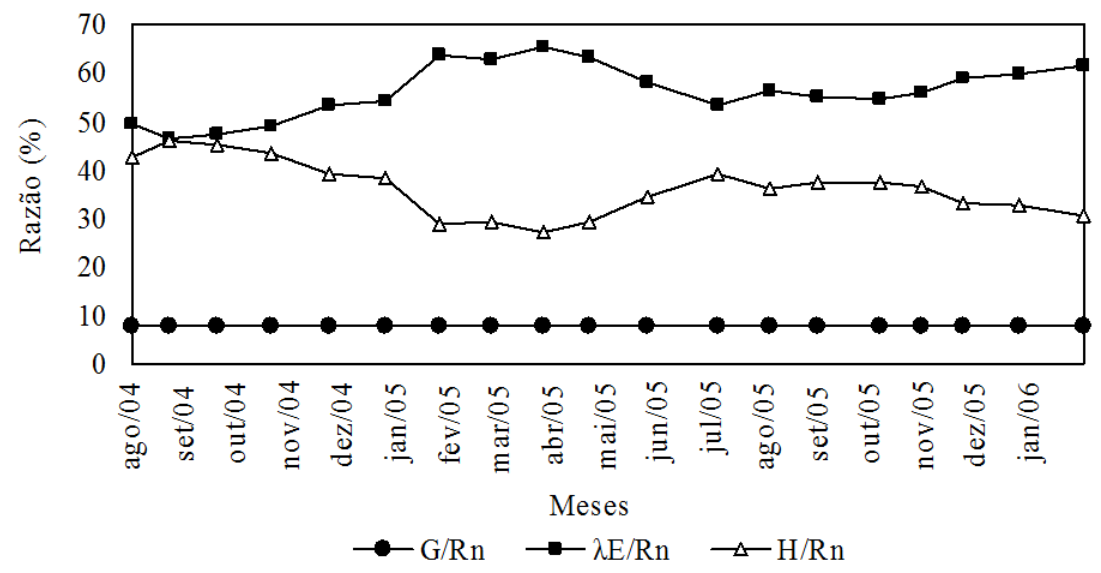

FIGURA 5: Variação sazonal da partição média mensal dos componentes do balanço de energia para o período estudado.

FIGURE 5: Seasonal variation of the average monthly partition of the energy balance components for the studied period.

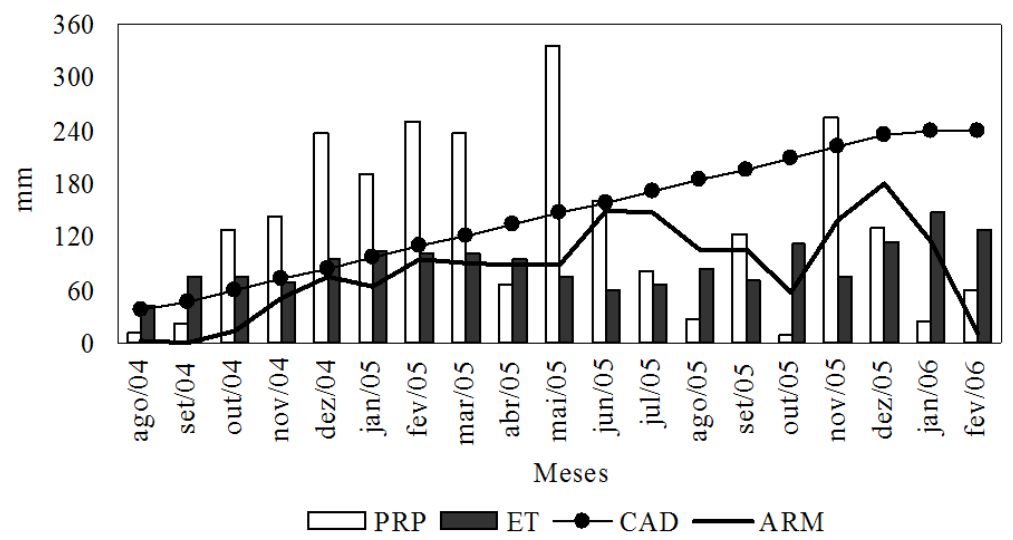

FIGURA 6: Precipitação, evapotranspiração, capacidade máxima de água disponível e armazenamento de água no solo ao longo do período estudado.

FIGURE 6: Precipitation, evapotranspiration, maximum amount of water available and storage of water in the soil during the studied period. 
$\mathrm{Na}$ maior parte o ARM foi menor que a CAD, somente nos meses de dezembro/04 e julho/05 é que ocorreu ARM máximo. Verifica-se que a partir de dezembro/05 houve estabilização da $\mathrm{CAD}$, pois o sistema radicular se estabeleceu nessa profundidade. Os valores encontrados para a CAD com variação do sistema radicular de 0,22 a $1,60 \mathrm{~m}$ variaram de 37,07 a $238,48 \mathrm{~mm}$ ao longo do período estudado. Sacramento Neto (2001), trabalhando com plantios jovens de eucalipto (1 e 2 anos) na região de Belo Oriente, na Bacia do Rio Doce, determinou que, em solos de baixada, a CAD variou de 137 a $171 \mathrm{~mm}$ e em solos de encosta, essa variação foi de 70 a 110 mm. Pereira et al. (2002), em espécies florestais a CAD varia de 150 a $300 \mathrm{~mm}$. O maior valor de ARM observado neste estudo foi de 180,23 mm. Souza (2006), em três regiões situadas na Bacia do Rio Doce em Minas Gerais, encontrou valores médios mensais de ARM de $79 \mathrm{~mm}$ em Belo Oriente, 119 mm em Ganhães e de 132 mm em Nova Era.

A Figura 7 mostra a variação diária do ARM observado e modelado ao longo do período. Observa-se que o comportamento do ARM obtido a partir do balanço hídrico utilizando a ET modelada, apresenta boa correspondência ao comportamento do ARM observado em campo durante o período de estudo. Isso pode ser confirmado pela correlação apresentada na Figura 8, em que se verifica um coeficiente de determinação de 0,94 e um coeficiente angular médio de 0,94 .

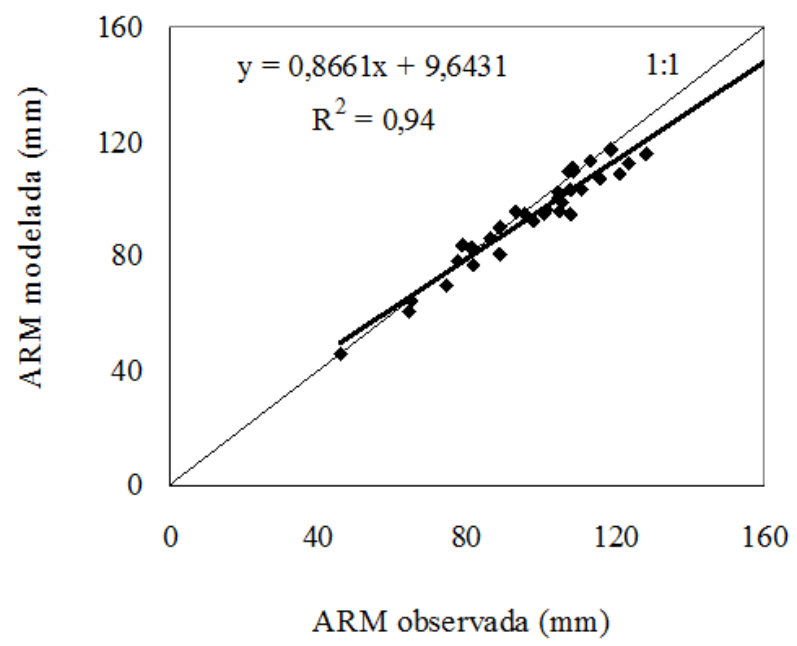

FIGURA 8: Relação entre a umidade do solo observada e a modelada.

FIGURE 8: Relationship between the observed and modeled soil moisture content.

\section{CONCLUSÃO}

Para esta fase de desenvolvimento do eucalipto e com as condições ambientais deste estudo, os valores de evapotranspiração das árvores foram menores do que em idades onde o terreno era completamente coberto pelo dossel vegetativo.

Tanto o método do balanço hídrico como o da Razão de Bowen foram úteis para a estimativa da evapotranspiração modelada.

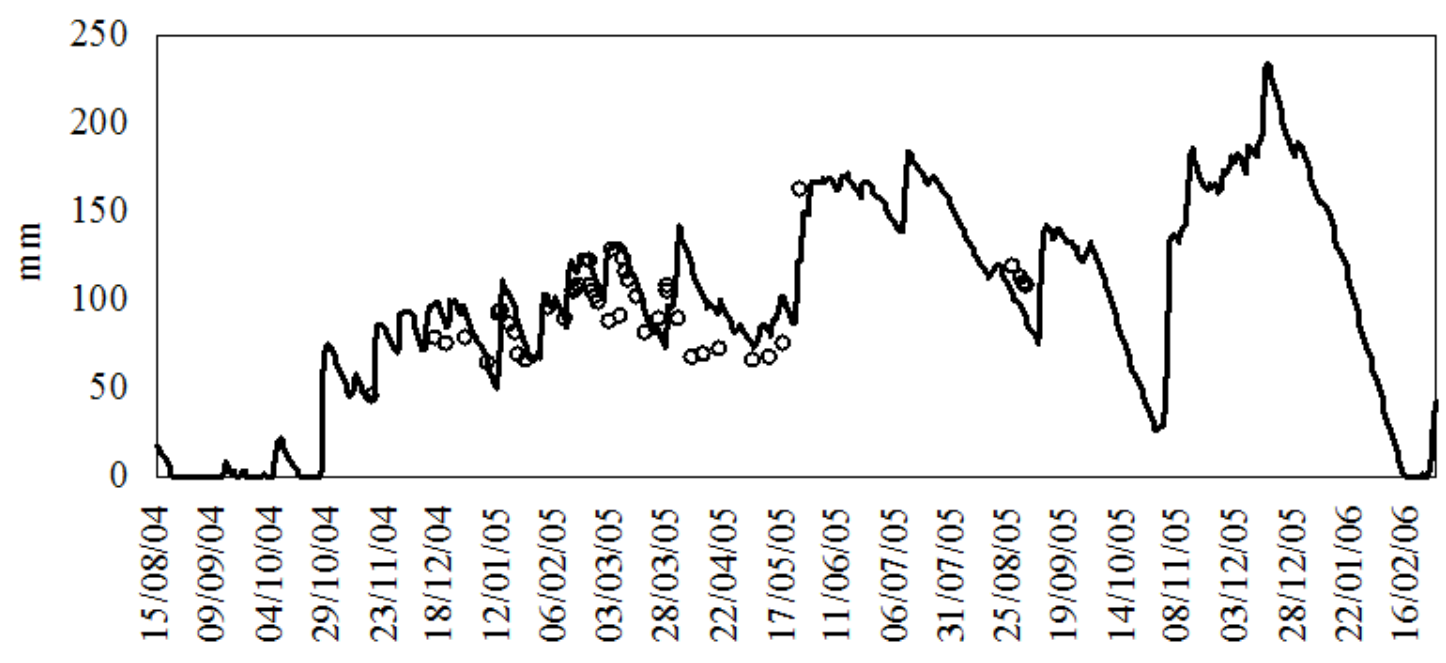

\section{Diário}

\section{— ARM modelado o ARM observado}

FIGURA 7: Evolução diária da umidade do solo observada e modelada.

FIGURE 7: Daily evolution of the soil moisture content both observed and modeled. 
Os dados de evapotranspiração obtidos pelo modelo mostraram-se coerentes com os resultados da evapotranspiração calculados pelo método da Razão de Bowen, justificando a modelagem feita para o eucalipto na fase inicial de desenvolvimento.

\section{REFERÊNCIAS BIBLIOGRÁFICAS}

ABRAF, Anuário Estatístico da Abraf 2010: Ano base 2009. Associação Brasileira de Produtores de Florestas Plantadas, Brasília, 2010.140 p.

ALMEIDA, A. C.et al. Mapping the effectof spatial and temporal variation in climate and soils on Eucalyptus plantationproduction with 3-PG, a process-based growth model. Forest Ecologyand Management, n. 259,p. 1730-1740, 2010.

ALMEIDA, A. C.et al. Growth and water balance of Eucalyptus grandis hybrid plantations in Brazil during a rotation for pulp production. Forest Ecology and Management, n. 251, p. 10-21, 2007.

BOWEN, I. S. The ratio of heat losses by conduction and by evaporation from any water surface. Physical Review, New York, n. 27, p. 779-787, 1926.

CABRAL, O. M. R. et al. The energy and water balance of a Eucalyptus plantation in southeast Brazil. Journal Hydrology, n. 388, p. 208-216, 2010.

DENMEAD, O. T.; BRADLEY, E. F. Flux-gradient relationships in a forest canopy.In: B.A. Hutchison and B.B. Hicks (Editors), The Forest-Atmosphere Interaction. Reidel, Dordrecht, 1985. p. 421-442.

DYE, P. J. Response of Eucalyptus grandis trees to soil waterdeficits. Tree Physiol, n. 16, p.233-238, 1996.

FORRESTER, D. I.; COLLOPY, J. J.; MORRIS, J. D. Transpiration along an age series of Eucalyptus globulus plantations in southeastern Australia. Forest Ecologyand Management, n. 259, p. 1754-1760, 2010.

HUBBARD, R. M. et al. Effects of irrigation on water use and water use efficiency in two fast growing eucalyptus plantations. Forest Ecology and Management, n. 259, p. 1714-1721, 2010.

LANDSBERG, J. J.; POWELL, D. B. B. Surface exchange characteristics of leaves subject to mutual interference. Agricultural and Forest Meteorology, n. 13, p. 169-184, 1973.

MIELKE, M. S. et al. Stomatal control of transpiration in the canopy of a clonal Eucalyptus grandisplantation. Trees, n. 13, p. 152-160. 1999.

MUNRO, D. S. Internal consistency of the Bowen ratio approach to flux estimation over forested wetland. In: B.A. Hutchison and B.B. Hicks (Editors). The Forest-Atmosphere Interaction. Reidel, Dordrecht, p. 395-406, 1985.

O'GRADY, A. P.; WORLEDGE, D.; BATTAGLIA, M. Constraints on transpiration ofEucalyptus globules in southern Tasmania, Australia. Agriculturaland Forest Meteorolgy, n. 148, p. 453-465, 2008.

PEREIRA, A. R.; ANGELOCCI, L. R.; SENTELHAS, P.C. Agrometeorologia: fundamentos e aplicações práticas. Guaíba: Agropecuária, 2002. $478 \mathrm{p}$.

RIOU, O.; VALANGOGNE, C.; PIERI, P. Un modèle simple d'interception du rayonnementsolairepar la vigne - vérificationexpérimentale. Agronomie, n. 9, p.441-450, 1989.

SACRAMENTO NETO, O. B. Balanço hídrico em plantios jovens de eucalipto na região de Belo Oriente-MG. 2001. 77 p., Dissertação (Mestrado)Universidade Federal de Viçosa, 2001.

SILBERSTEIN, R. P. et al.Energy balance of a natural jarrah (Eucalyptus marginata) forest in Western Australia. Measurements during spring and summer. Agricultural and Forest Meteorology, n. 109, p. 79-104, 2001.

SOUZA, M. J. H. et al. Disponibilidade hídrica do solo e produtividade do eucaliptoem três regiões da bacia do Rio Doce. Revista Árvore,Viçosa, v.30, n.3, p.399-410, 2006.

SOUZA, W. G. Modelagem da evapotranspiração em plantios jovens de eucalipto em fase inicial de desenvolvimento com cobertura parcial do solo. 2006., 40 p., Dissertação (Mestrado) - Universidade Federal de Viçosa, 2006.

THORPE, M. R.; WARRIT, B.; LANDSBERG, J. J. Responses of apple leat stomata: a model for single leaves and a whole tree. Plant, Cell and Environment, n. 3, p. 23-27, 1980.

XAVIER, A. C.; SOARES, J. V.; ALMEIDA, A. C. Variação do índice de área foliar em clones de eucalipto ao longo do seu ciclo de crescimento. Revista Árvore, v. 26, n. 4, p. 421- 427, 2002.

YUNUSA, I.A.M. et al. Topographical and seasonal trends in transpiration by two co-occurring Eucalyptus species during two contrasting years in a low rainfall environment. Agricultural and Forest Meteorology, n. 150, p. 1234 - 1244, 2010.

ZANON, M. L. B.; FINGER, C. A. G. Relação de variáveis meteorológicas com o crescimento das árvores de Araucaria angustifolia (bertol.) Kuntze em povoamentos implantados. Ciência Florestal, Santa Maria, v. 20, n. 3, p. 467-476, 2010. 